\title{
A Comprehensive Presentation of the Turbulent Plane Jet Theory with Passive Scalar
}

\author{
D. Violeau \\ EDF Lab, 6 quai Watier, 78400 Chatou, France \\ Correspondence should be addressed to D. Violeau; damien.violeau@edf.fr
}

Received 18 December 2016; Accepted 23 April 2017; Published 25 May 2017

Academic Editor: Rama S. R. Gorla

Copyright (C) 2017 D. Violeau. This is an open access article distributed under the Creative Commons Attribution License, which permits unrestricted use, distribution, and reproduction in any medium, provided the original work is properly cited.

We present a unified vision of the existing theoretical models for the turbulent plane jet, leading to new analytical profiles for scalar concentration and turbulent quantities, including a complete turbulent kinetic energy budget. Integrals of the budget terms are also computed. The present model is split into two variants. Both compare fairly well with referenced experimental data.

\section{Introduction}

The theory of the turbulent planar jet has already motivated a lot of publications. Pope [1] made a thorough description of the standard theoretical approach based on the boundary layer approximation and self-similarity, leading to a theoretical profile for the velocity field. This method, based on mass and momentum conservation laws, has been extensively discussed (see, e.g., [2-4]) and is in good agreement with experiments except far away from the jet center part where it overestimates the axial jet velocity field $[1,2]$. On the other hand, some authors prefer using a purely empirical Gaussian profile [5-8] which provides a better fit to experimental data. Theoretical profiles for the turbulent quantities such as eddy viscosity (as far as this concept is admitted) and the various terms of the turbulent kinetic energy (TKE) budget have also been provided $[1,8]$ and compared to the experimental data obtained by Bradbury [2], Heskestad [9], Gutmark and Wygnanski [10], Ramapriyan and Chandrasekhara [11], among others. However-to our knowledge-there exists no publication giving a complete and consistent method leading to theoretical approximated profiles for all these quantities. As an example, Agrawal and Prasad [8] give theoretical formulae for Reynolds stresses, TKE production, and eddy viscosity from the Gaussian velocity assumption, but they do not provide any experimental validation. Finally, the distribution of nonbuoyant scalar concentration in a plane jet has been poorly addressed; Kotsovinos and List [5] suggest a Gaussian empirical profile while Law [12] gives a theoretical formula based on scalar flux conservation, but his mathematical expression is cumbersome and requires a function defined in the complex plane.

In this paper, we first build a theoretical formalism that allows treating all transported quantities in a similar way, on the assumptions of boundary layer approximation and self-similarity. In particular, conserved quantities (i.e., momentum and scalar concentration) are treated on the same foot, leading to unified dimensionless governing equations and solutions. The solutions are obtained as the product of two terms: a power law for the axial dimensionless coordinate $\zeta$ and a function of the dimensionless transverse coordinate $\xi$ that is determined by solving the governing equations. With this method, the standard velocity profile is recovered while a new analytical profile for scalar concentration is given. Other quantities like TKE and dissipation rate are searched in the same generic form and substituted in the $k-\epsilon$ governing equations, giving their dependency to $\zeta$. By assuming a production-to-dissipation equilibrium, analytical formulae are then obtained for all quantities of interest, including the TKE budget terms and Taylor microscale. The latter method is then applied to the Gaussian empirical velocity profile, giving a second variant of this theory. It is found that all present analytical formulae are in good agreement with experimental observations except some of them (dissipation, convection, and diffusion of TKE) in the center part of the jet. The present approach thus provides a unified way to 


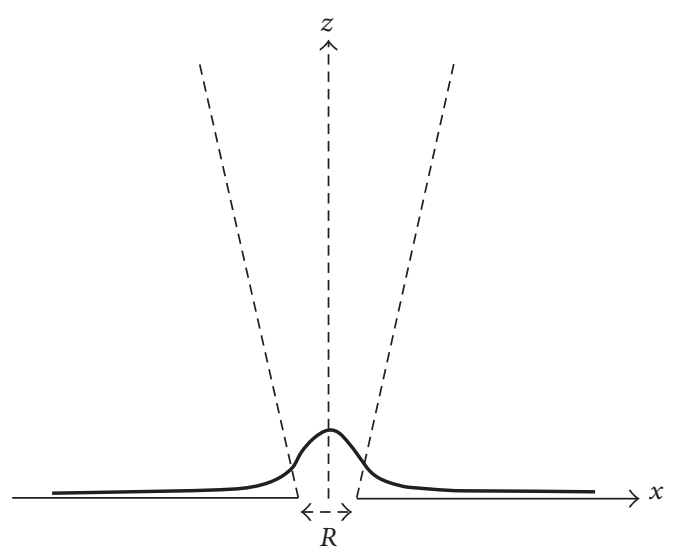

FIgURE 1: Notation. The curve shows a typical axial velocity profile.

understanding the (approximate) theory of plane turbulent, self-preserving jet.

\section{Theory}

2.1. State of the Art and Notation. We consider a steady incompressible plane turbulent jet determined by an infinite orifice (or slot) of width $R$ (see Figure 1). We work in the $(x, z)$ plane, with $z$ being the coordinate along the axis of the jet, $v$ the fluid molecular kinematic viscosity, and $K$ the scalar molecular diffusion coefficient. The spatial distribution of (Reynolds-averaged) longitudinal (or axial) fluid velocity $\bar{w}$ and scalar concentration $\bar{c}$ will be searched as function of the fluid discharge velocity and concentration $U$ and $C$.

In the sequel, we assume the molecular fluxes and stresses to be negligible with respect to turbulent fluxes and stresses, so that the Reynolds and Schmidt numbers have no effect. We define the vector $\mathbf{A}=(\bar{w}, \bar{c})^{T}$ of conserved quantities and, assuming Prandlt's boundary layer assumptions to be valid, write the governing equations as

$$
\begin{aligned}
\frac{\partial \bar{u}}{\partial x}+\frac{\partial \bar{w}}{\partial z} & =0, \\
\frac{\partial \bar{w} \mathbf{A}}{\partial z} & =-\frac{\partial}{\partial x}\left(\bar{u} \mathbf{A}+\overline{u^{\prime} \mathbf{A}^{\prime}}\right),
\end{aligned}
$$

where primes refer to turbulent fluctuations and overbars to Reynolds-averaging. The symmetry condition through the $z$ axis implies

$$
\begin{aligned}
\bar{u}(0, z) & =0 \\
\frac{\partial \bar{w}}{\partial x}(0, z) & =0 \\
\frac{\partial \bar{c}}{\partial x}(0, z) & =0 .
\end{aligned}
$$

On the other hand, the boundary conditions far away from the jet are vanishing of axial velocity and concentration, as well as turbulent shear stress and scalar flux:

$$
\begin{aligned}
\lim _{x \rightarrow \pm \infty} \bar{w}(x, z) & =0 \\
\lim _{x \rightarrow \pm \infty} \overline{u^{\prime} w^{\prime}}(x, z) & =0 \\
\lim _{x \rightarrow \pm \infty} \bar{c}(x, z) & =0 . \\
\lim _{x \rightarrow \pm \infty} \overline{u^{\prime} c^{\prime}}(x, z) & =0
\end{aligned}
$$

Experiments (e.g., Bradbury, 1965; see the review by Pope, 2000) show that the plane jet half-width $x^{*}(z)$ is a linear function of $z$. As in the classical theory of the turbulent plane jet without a scalar, we thus define

$$
\begin{aligned}
\xi & =\frac{x}{x^{*}(z)} \\
\zeta & =\frac{z-z_{0}}{R},
\end{aligned}
$$

where $z_{0}$ is a virtual origin. $x /\left(z-z_{0}\right)$ is thus a linear function of $\xi$ and assumes autosimilarity of the jet. We denote by $S \doteqdot$ $d x^{*} / d z$ the slope of the half-width.

Then, it is known that integrating the momentum and scalar governing equations along the cross-stream direction gives

$$
\begin{aligned}
& \frac{\bar{w}}{U}=\frac{\alpha_{w}}{\zeta^{1 / 2}} f_{w}(\xi) \\
& \overline{\bar{c}}=\frac{\alpha_{c}}{\zeta^{1 / 2}} f_{c}(\xi) .
\end{aligned}
$$

For the two unknown functions $f_{i}$, we may impose $f_{i}(0)=1$ for each $i \in\{w, c\}$, since the dimensionless constants $\alpha_{i}$ are unknown. The above dependencies in $\zeta^{-1 / 2}$ are in agreement with Kotsvinos and List [5] and Pope [1], as well as with Gutmark and Wygnanski's [10] measurements.

2.2. Advection and Diffusion. We now investigate the advection and diffusion of an arbitrary field $A$ in the following generic form (in most cases the power $\beta_{A}$ will be set to $1 / 2$ later on, according to the previous subsection):

$$
A(x, z)=\frac{\alpha_{A} A_{0}}{\zeta_{A}} f_{A}(\xi)
$$

where $A_{0}$ is a dimensional constant ( $U$ or $C$ in the present case). The purpose of the next developments is to write the governing equations (momentum, scalar, and turbulent quantities) in a unified form. We get

$$
\begin{aligned}
& \frac{\partial A}{\partial x}=\frac{\alpha_{A} A_{0}}{S R \beta^{\beta_{A}+1}} f_{A}^{\prime}(\xi) \\
& \frac{\partial A}{\partial z}=-\frac{\alpha_{A} A_{0}}{R \zeta \beta_{A}+1}\left[\beta_{A} f_{A}(\xi)+\xi f_{A}^{\prime}(\xi)\right]
\end{aligned}
$$


(here and in the sequel, the prime denotes differentiation with respect to $\xi$, except in the turbulent fluctuations $u^{\prime}$, etc.). Using (1) and applying (9) to $A=\bar{w}$ with $\beta_{w}=1 / 2$ we get

$$
\begin{aligned}
\frac{\partial \bar{u}}{\partial x} & =-\frac{\partial \bar{w}}{\partial z} \\
& =\frac{\alpha_{w} U}{R \zeta^{3 / 2}}\left[\frac{1}{2} f_{w}(\xi)+\xi f_{w}^{\prime}(\xi)\right] .
\end{aligned}
$$

We now define the antiderivative of $f_{w}$ as

$$
F_{w}(\xi)=\int_{0}^{\xi} f_{w}\left(\xi^{\prime}\right) d \xi^{\prime}
$$

with $F_{w}(0)=0$. Thus, (11) gives the transverse dimensionless jet velocity as

$$
\frac{\bar{u}}{U}=\frac{S \alpha_{w}}{\zeta^{1 / 2}}\left[\xi f_{w}(\xi)-\frac{1}{2} F_{w}(\xi)\right]
$$

From that, we can compute the advection of the arbitrary field $A$ :

$$
\begin{aligned}
\frac{\partial \bar{u} A}{\partial x} & +\frac{\partial \bar{w} A}{\partial z} \\
= & -\frac{\alpha_{w} \alpha_{A} U A_{0}}{R \zeta^{\beta_{A}+3 / 2}}\left[\beta_{A} f_{w}(\xi) f_{A}(\xi)+\frac{1}{2} F_{w}(\xi) f_{A}^{\prime}(\xi)\right] \\
= & -\frac{\alpha_{w} \alpha_{A} U A_{0}}{R \zeta^{\beta_{A}+3 / 2}} \frac{f_{A}^{1 / 2}(\xi)\left(F_{w}^{\beta_{A}} f_{A}^{1 / 2}\right)^{\prime}(\xi)}{F_{w}^{\beta_{A}-1}(\xi)}
\end{aligned}
$$

For the two components of the vector $\mathbf{A}$ defined above (axial velocity and concentration), since the powers are equal to $1 / 2$ we find

$$
\frac{\partial \bar{u} A_{i}}{\partial x}+\frac{\partial \bar{w} A_{i}}{\partial z}=-\frac{\alpha_{w} \alpha_{i} U A_{i 0}}{2 R \zeta^{2}}\left(F_{w} f_{i}\right)^{\prime}(\xi)
$$

with $A_{w 0}=U$ and $A_{c 0}=C$.

We now address the diffusion of $A$. We model the turbulent flux and stress with the eddy viscosity assumption:

$$
\begin{aligned}
& \overline{u^{\prime} w^{\prime}}=-v_{T} \frac{\partial \bar{w}}{\partial x} \\
& \overline{u^{\prime} c^{\prime}}=-\frac{\nu_{T}}{\sigma_{c}} \frac{\partial \bar{c}}{\partial x},
\end{aligned}
$$

where $\sigma_{c}$ is the turbulent Schmidt number. We can put these two formulae together by defining $\sigma_{i}, i \in\{w, c\}$ where $\sigma_{w}=$ 1. Now, from (6) the diffusion terms in (2) read

$$
\begin{aligned}
-\frac{\partial \overline{u^{\prime} A_{i}^{\prime}}}{\partial x} & =\frac{\partial}{\partial x}\left(\frac{\nu_{T}}{\sigma_{i}} \frac{\partial A_{i}}{\partial x}\right) \\
& =\frac{\alpha_{i}}{S^{2} \sigma_{i}} \frac{A_{i 0}}{R^{2}} \frac{1}{\zeta^{5 / 2}} \frac{\partial}{\partial \xi}\left[\nu_{T}(\xi, \zeta) f_{i}^{\prime}(\xi)\right] .
\end{aligned}
$$

According to (3) and (4), the dimensionless unknown functions introduced so far are subject to the following boundary conditions:

$$
\begin{aligned}
\lim _{\xi \rightarrow \pm \infty} F_{w}^{\prime}(\xi) & =0 \\
\lim _{\xi \rightarrow \pm \infty} F_{w}^{\prime \prime}(\xi) & =0 \\
F_{w}(0) & =0 \\
F_{w}^{\prime \prime}(0) & =0, \\
\lim _{\xi \rightarrow \pm \infty} f_{c}(\xi) & =0 \\
\lim _{\xi \rightarrow \pm \infty} f_{c}^{\prime}(\xi) & =0 \\
f_{c}^{\prime}(0) & =0
\end{aligned}
$$

with the arbitrary additional conditions $F_{w}^{\prime}(0)=1$ and $f_{c}(0)=1$. Now, the momentum and concentration equations (2) can be rewritten using (16) and (19) and integrating once with respect to $\xi$ :

$$
F_{w}(\xi) f_{i}(\xi)=-\frac{2}{S^{2} \sigma_{i} \alpha_{w} U R} \frac{\nu_{T}(\xi, \zeta)}{\zeta^{1 / 2}} f_{i}^{\prime}(\xi),
$$

where $i \in\{w, c\}$.

2.3. Turbulent Closure. Equation (22) tells us that the eddy viscosity should depend on $\zeta$ as $\zeta^{1 / 2}$ in the turbulent plane jet, as already known [1]. This is in agreement with the generic form (7) with $\beta_{\gamma_{T}}=-1 / 2$ and $A_{\gamma_{T}}=U R$ :

$$
\frac{\nu_{T}}{U R}=\frac{\alpha_{\nu_{T}}}{\zeta^{-1 / 2}} f_{\nu_{T}}(\xi)
$$

Thus, (22) gives

$$
F_{w}(\xi) f_{i}(\xi)+\frac{2 \alpha_{\nu_{T}}}{\sigma_{i} \alpha_{w}} f_{\nu_{T}}(\xi) f_{i}^{\prime}(\xi)=0
$$

$(i \in\{w, c\})$. We may first try to compute the eddy viscosity from the standard $k-\epsilon$ model; that is, $\nu_{T}=C_{\mu}\left(k^{2} / \epsilon\right)$. Under the steady boundary layer assumptions, the TKE and dissipation rate governing equations read

$$
\begin{aligned}
& \underbrace{\frac{\partial \bar{u} k}{\partial x}+\frac{\partial \bar{w} k}{\partial z}}_{-C_{k}}=\underbrace{C_{\mu} \frac{k^{2}}{\epsilon}\left(\frac{\partial \bar{w}}{\partial x}\right)^{2}}_{P_{k}}+\underbrace{\frac{C_{\mu}}{\sigma_{k}} \frac{\partial}{\partial x}\left(\frac{k^{2}}{\epsilon} \frac{\partial k}{\partial x}\right)}_{D_{k}}-\epsilon \\
& \frac{\partial \bar{u} \epsilon}{\partial x}+\frac{\partial \bar{w} \epsilon}{\partial z}= C_{\mu} C_{\epsilon 1} k\left(\frac{\partial \bar{w}}{\partial x}\right)^{2}+\frac{C_{\mu}}{\sigma_{\epsilon}} \frac{\partial}{\partial x}\left(\frac{k^{2}}{\epsilon} \frac{\partial \epsilon}{\partial x}\right) \\
&-C_{\epsilon 2} \frac{\epsilon^{2}}{k}
\end{aligned}
$$

with $C_{\mu}=0.09, \sigma_{k}=1.0, \sigma_{\epsilon}=1.3, C_{\epsilon 1}=1.44$, and $C_{\epsilon 2}=$ 1.92 [14]. Contrary to the momentum and concentration, we 
cannot write conserved fluxes of $k$ and $\epsilon$ because of source terms in the governing equations (25) and (26). However, as for the other unknowns, we may seek for $k$ and $\epsilon$ in the following form:

$$
\begin{aligned}
\frac{k}{U^{2}} & =\frac{\alpha_{k}}{\zeta \beta_{k}} f_{k}(\xi) \\
\frac{\epsilon}{U^{3} / R} & =\frac{\alpha_{\epsilon}}{\zeta \beta_{\epsilon}} f_{\epsilon}(\xi),
\end{aligned}
$$

where $\alpha_{k}$ and $\alpha_{\epsilon}$ are two unknown dimensionless constants. Using the general relations (16) and (19), the substitution of these models into (25) and (26) gives

$$
\begin{aligned}
- & \frac{\alpha_{w} \alpha_{k}}{\zeta \beta_{k}+3 / 2} \frac{f_{k}^{1 / 2}\left(F_{w}^{\beta_{k}} f_{k}^{1 / 2}\right)^{\prime}}{F_{w}^{\beta_{k}-1}} \\
= & \frac{C_{\mu} \alpha_{w}^{2} \alpha_{k}^{2}}{S^{2} \alpha_{\epsilon} \zeta^{2 \beta_{k}-\beta_{\epsilon}+3}} \frac{f_{w}^{\prime 2} f_{k}^{2}}{f_{\epsilon}} \\
& +\frac{C_{\mu} \alpha_{k}^{3}}{S^{2} \sigma_{k} \alpha_{\epsilon} \zeta^{3 \beta_{k}-\beta_{\epsilon}+2}}\left(\frac{f_{k}^{2} f_{k}^{\prime}}{f_{\epsilon}}\right)^{\prime}-\frac{\alpha_{\epsilon}}{\zeta^{\beta_{\epsilon}}} f_{\epsilon} \\
-\frac{\alpha_{w} \alpha_{\epsilon}}{\zeta \beta_{\epsilon}+3 / 2} \frac{f_{\epsilon}^{1 / 2}\left(F_{w}^{\beta_{\epsilon}} f_{\epsilon}^{1 / 2}\right)^{\prime}}{F_{w}^{\beta_{\epsilon}-1}} & \\
= & \frac{C_{\mu} C_{\epsilon 1} \alpha_{w}^{2} \alpha_{k}}{S^{2} \zeta^{\beta_{k}+3}} f_{w}^{\prime 2} f_{k}+\frac{C_{\mu} \alpha_{k}^{2}}{S^{2} \sigma_{\epsilon} \zeta^{2 \beta_{k}+2}}\left(\frac{f_{k}^{2} f_{\epsilon}^{\prime}}{f_{\epsilon}}\right)^{\prime} \\
& -\frac{C_{\epsilon 2} \alpha_{\epsilon}^{2}}{\alpha_{k} \zeta^{2 \beta_{\epsilon}-\beta_{k}}} \frac{f_{\epsilon}^{2}}{f_{k}}
\end{aligned}
$$

(we dropped the explicit dependency of all functions on $\xi$ for the sake of clarity). The dependency on $\zeta$ should cancel in both equations, which is possible if and only if $\beta_{k}=1$ and $\beta_{\epsilon}=5 / 2$. Hence, the TKE in the jet is inversely proportional to the dimensionless distance from the jet virtual origin $\zeta$, in agreement with Gutmark and Wygnanski [10]. With the relation giving $\nu_{T}$ as a function of $k$ and $\epsilon$, this gives us

$$
\frac{\nu_{T}}{U R}=\frac{C_{\mu} \alpha_{k}^{2}}{\alpha_{\epsilon}} \zeta^{1 / 2} \frac{f_{k}^{2}(\xi)}{f_{\epsilon}(\xi)}
$$

in agreement with (23) with $f_{v_{T}}=f_{k}^{2} / f_{\epsilon}$. Note that the dependency of $k$ and $\epsilon$ on $\xi$ gives the size of the large turbulent eddies (or integral scale) $L_{t}=C_{\mu}^{3 / 4} k^{3 / 2} / \epsilon$ as

$$
\frac{L_{t}}{R}=\frac{C_{\mu}^{3 / 4} \alpha_{k}^{3 / 2}}{\alpha_{\epsilon}} \zeta \frac{f_{k}^{3 / 2}(\xi)}{f_{\epsilon}(\xi)}
$$

Thus, the large turbulent eddy size on the jet axis is proportional to the distance to the origin of the jet, in agreement with observations [1]. We now set

$$
\gamma_{i} \doteqdot \frac{4 C_{\mu} \alpha_{k}^{2}}{S^{2} \sigma_{i} \alpha_{w} \alpha_{\epsilon}}
$$

for $i \in\{w, c, k, \epsilon\}$. Then, the momentum and concentration equations read

$$
F_{w} f_{i}+\frac{\gamma_{i}}{2} \frac{f_{k}^{2} f_{i}^{\prime}}{f_{\epsilon}}=0
$$

for $i \in\{w, c\}$, while the $k$ - $\epsilon$ equations become

$$
\begin{aligned}
& f_{w} f_{k}+\frac{1}{2} F_{w} f_{k}^{\prime}+\frac{C_{\mu} \alpha}{S^{2}} \frac{f_{w}^{\prime 2} f_{k}^{2}}{f_{\epsilon}}+\frac{\gamma_{k}}{4}\left(\frac{f_{k}^{2} f_{k}^{\prime}}{f_{\epsilon}}\right)^{\prime} \\
& -\frac{1}{\alpha} f_{\epsilon}=0 \\
& \frac{5}{2} f_{w} f_{\epsilon}+\frac{1}{2} F_{w} f_{\epsilon}^{\prime}+\frac{C_{\mu} C_{\epsilon 1} \alpha}{S^{2}} f_{w}^{\prime 2} f_{k}+\frac{\gamma_{\epsilon}}{4}\left(\frac{f_{k}^{2} f_{\epsilon}^{\prime}}{f_{\epsilon}}\right)^{\prime} \\
& -\frac{C_{\epsilon 2}}{\alpha} \frac{f_{\epsilon}^{2}}{f_{k}}=0,
\end{aligned}
$$

where

$$
\alpha \doteqdot \frac{\alpha_{w} \alpha_{k}}{\alpha_{\epsilon}}
$$

Equations (33) and (34) should be provided with boundary conditions identical to (21). Since the dimensionless constants $\alpha_{k}$ and $\alpha_{\epsilon}$ have not yet been specified, we may choose $f_{k}(0)=f_{\epsilon}(0)=1$.

\section{Solutions and Validation}

3.1. Velocity and Scalar Profiles. The system given by (32), (33), and (34) is a nonlinear coupled system of four unknown functions (recall (32) contains two equations), and it looks hard to find analytical solutions. Similar systems have been proposed to study round jets and plumes [15] and then solved by numerical integration. In the present case, the theory of plane jet without any scalar assumes that turbulent quantities do not depend on the horizontal dimensionless coordinate $\xi$ [1]. This is obviously a very coarse hypothesis, since the turbulence is mainly confined within the jet. However, having no method to solve the complete abovementioned system, we will keep this assumption as a first approximation. In other words, we assume $f_{k}^{2}(\xi)=f_{\epsilon}(\xi)$, in contradiction with Bradbury's [2] experiments (a more advanced assumption will be presented in Section 3.2). This crude assumption is known to provide a decent velocity profile. It is easy to see that it is also in disagreement with (33) and (34). Thus, in this first approach the latter two equations are dropped and we only need to solve (32), which now reads

$$
\begin{gathered}
F_{w} F_{w}^{\prime}+\frac{\gamma_{w}}{2} F_{w}^{\prime \prime}=0 \\
F_{w} f_{c}+\frac{\gamma_{c}}{2} f_{c}^{\prime}=0 .
\end{gathered}
$$


With the boundary conditions mentioned above, (36) has the well-known solution for the self-similar distribution of the jet axial velocity (see, e.g., [1]):

$$
\begin{aligned}
& F_{w}(\xi)=\delta \tanh \frac{\xi}{\delta} \\
& f_{w}(\xi)=F_{w}^{\prime}(\xi)=\operatorname{sech}^{2} \frac{\xi}{\delta}
\end{aligned}
$$

with $\delta \doteqdot \sqrt{\gamma_{w}}$. Then, (37) can be readily solved with its boundary conditions and using the definition (31) to give

$$
f_{c}(\xi)=\operatorname{sech}^{2 \sigma_{c}} \frac{\xi}{\delta} \text {. }
$$

Finally, the distribution of axial velocity and concentration can be synthetized in the following formula:

$$
\frac{A_{i}(\xi, \zeta)}{A_{i 0}}=\frac{\alpha_{i}}{\zeta^{1 / 2}} \operatorname{sech}^{2 \sigma_{i}} \frac{\xi}{\delta}
$$

(recall $A_{w}=\bar{u}$ and $A_{c}=\bar{c}$, by definition of the vector $\mathbf{A}$, with $A_{w 0}=U$ and $\left.A_{c 0}=C\right)$. In a more comprehensive way, the final formulae for axial velocity and concentration are

$$
\begin{aligned}
& \frac{\bar{w}(\xi, \zeta)}{U}=\frac{\alpha_{w}}{\sqrt{\zeta}} \operatorname{sech}^{2} \frac{\xi}{\delta} \\
& \frac{\bar{c}(\xi, \zeta)}{C}=\frac{\alpha_{c}}{\sqrt{\zeta}} \operatorname{sech}^{2 \sigma_{c}} \frac{\xi}{\delta} .
\end{aligned}
$$

The constants can be deduced by integrating the fluxes $\bar{w} \mathbf{A}$ over the cross-stream directions and using $d x=$ $x^{*}(z) d \xi=S R \zeta d \xi$, which gives

$$
\alpha_{w} \alpha_{i} J_{i}=\frac{Q_{i}^{*}}{S}
$$

where

$$
J_{i} \doteqdot \int_{-\infty}^{+\infty} f_{w}(\xi) f_{i}(\xi) d \xi
$$

$(i=w, c)$. The dimensionless fluxes of momentum and scalar $Q_{i}^{*}$ can be calculated upstream of the orifice. We may assume the Reynolds-averaged velocity to have a log-profile and the scalar concentration to be uniformly distributed along the pipe section, giving $Q_{w}^{*} \approx Q_{c}^{*}=1$. Then we compute the integrals $J_{i}$ :

$$
J_{i}=\int_{-\infty}^{+\infty} \operatorname{sech}^{2\left(1+\sigma_{i}\right)} \frac{\xi}{\delta} d \xi=\frac{2 \sqrt{\pi} \delta}{1+2 \sigma_{i}} \frac{\Gamma\left(1+\sigma_{i}\right)}{\Gamma\left(1 / 2+\sigma_{i}\right)},
$$

where $\Gamma$ denotes Euler's function of first kind. We finally get

$$
\begin{aligned}
& \alpha_{w}=\frac{1}{2} \sqrt{\frac{3}{S \delta}} \\
& \alpha_{c}=\frac{1+2 \sigma_{c}}{\sqrt{3 \pi S \delta}} \frac{\Gamma\left(1 / 2+\sigma_{c}\right)}{\Gamma\left(1+\sigma_{c}\right)} .
\end{aligned}
$$

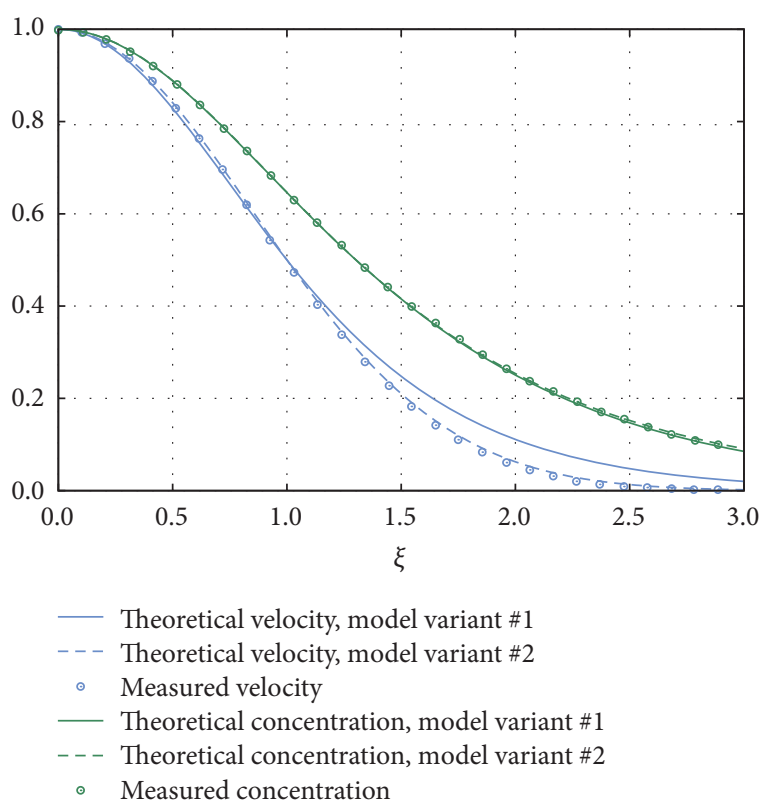

Figure 2: Profiles of axial velocity (blue) and concentration (green). Present models \#1 (see (41) and (42), solid lines) and \#2 (see (52) and (54), dashed lines) versus measurements (symbols) from Fischer et al. [13].

Note that (31) and (46) give

$$
\begin{aligned}
\frac{\alpha_{k}^{2}}{\alpha_{\epsilon}} & =\frac{9}{64 C_{\mu} \alpha_{w}^{3}} \\
& =\frac{\sqrt{3}(S \delta)^{3 / 2}}{8 C_{\mu}} .
\end{aligned}
$$

We can see that $S$ and $\delta$ appear everywhere through their product, which is a natural consequence of the arbitrary definition of the jet slope. Moreover, the profiles (41) and (42) depend on $\xi / \delta$, as all subsequent functions will do. In the sequel, we will thus use the following notations:

$$
\begin{aligned}
& \phi \doteqdot S \delta \\
& \eta \doteqdot \frac{\xi}{\delta} .
\end{aligned}
$$

We now determine the model constants. By definition of the jet half-width we have $\bar{w}(1, \zeta)=(1 / 2) \bar{w}(0, \zeta)$; therefore, (41) gives us

$$
\delta=\frac{1}{\ln (1+\sqrt{2})} \approx 1.135
$$

Figure 2 shows the present model for the velocity and scalar concentration profiles (41) and (42) for $S=0.0970$, which is in agreement with experimental data (e.g., [1, $2,9,10])$. With that, (49) yields $\phi \approx 0.1101$. For the concentration profile, $\sigma_{c}=0.63$ was chosen in order to fit the data, while other authors recommend $\sigma_{c}=0.71$ ([16], 
experimental), 0.72 ([17], theoretical), 0.75 ([18], theoretical), or 0.74 (Violeau, 2009, theoretical) for simple shear flows. On the other hand, Law [12] suggests $\sigma_{c}=0.59$ for the theory of the plane jet, but his concentration profile is in a modest agreement with experimental data.

With the present values, (46) and (47) yield $\alpha_{w} \approx 2.610$ and $\alpha_{c} \approx 2.325$. One can see the model for the concentration distribution is in a very good agreement with Fischer et al.s [13] data. It should be noted that our solution (39) matches exactly the theoretical profile proposed by Law [12], although the latter was presented in a much more complicated way involving a function of complex variables.

As for the velocity profile, another formula is used by several authors (e.g., [5-8]), which is purely empirical:

$$
f_{w}(\eta)=e^{-\eta^{2}} \text {. }
$$

With the above Gaussian profile, the definition of the jet half-width gives

$$
\delta=\frac{1}{\sqrt{\ln 2}} \approx 1.201
$$

in place of $(51)$. With the abovementioned value of $S$, we now have $\phi \approx 0.1165$. Figure 2 shows that (52) fits the experimental velocity profile better than (41) away from the center part of the jet.

Using this new velocity profile to calculate $F_{w}$ and solving (37) for the concentration give another theoretical profile:

$$
f_{c}(\eta)=\exp \left[-\sigma_{c}\left(e^{-\eta^{2}}+\sqrt{\pi} \eta \operatorname{erf} \eta-1\right)\right] .
$$

The integrals $J_{i}$ also have new values, and (43) gives

$$
\begin{aligned}
\alpha_{w} & =\left(\frac{2}{\pi \phi^{2}}\right)^{1 / 4} \approx 2.617 \\
\alpha_{c} & =\left(\frac{\pi}{2 \phi^{2}}\right)^{1 / 4} \\
& \cdot\left[\int_{-\infty}^{+\infty} e^{-\eta^{2}} \exp \left[-\sigma_{c}\left(e^{-\eta^{2}}+\sqrt{\pi} \eta \operatorname{erf} \eta_{1}\right)\right] d \eta\right]^{-1} \\
& \approx 2.350 .
\end{aligned}
$$

The integral (56) giving $\alpha_{c}$ was obtained numerically using $\sigma_{c}=0.70$. The latter value was set in order to fit Fischer et al.s [13] experimental results for the concentration (Figure 2). It can be seen that (39) and (54) are graphically very close to each other (for these values of $\sigma_{c}$, that are not the same in the two models).

3.2. Turbulent Quantities. We now want to provide analytical formulae for the turbulent quantities. Assuming a production-to-dissipation equilibrium in the TKE equation (25), that is, $P_{k}=\epsilon$, the corresponding terms in (33) (3rd and last terms) give

$$
f_{\epsilon}=-\frac{\sqrt{C_{\mu}} \alpha}{S} f_{w}^{\prime} f_{k} .
$$

With the momentum equation (22) (for $i=w$ ), relation (29), and definitions (31) and (35), we get

$$
\begin{gathered}
\alpha_{k} f_{k}=\frac{S \alpha_{w}^{2}}{2 \sqrt{C_{\mu}}} F_{w} f_{w} \\
\alpha_{\epsilon} f_{\epsilon}=-\frac{\alpha_{w}^{3}}{2} F_{w} f_{w} f_{w}^{\prime}
\end{gathered}
$$

(note that $f_{w}^{\prime} \leq 0$ ). Only the product $\alpha_{k} f_{k}$ and $\alpha_{\epsilon} f_{\epsilon}$ can be predicted, since we arbitrarily set $f_{k}(0)=f_{\epsilon}(0)=1$. With (29) again and (41), relation (46), and definition (50), we get

$$
\begin{aligned}
& \alpha_{k} f_{k}(\eta)=\frac{3}{8 \sqrt{C_{\mu}}} \frac{\sinh \eta}{\cosh ^{3} \eta} \\
& \alpha_{\epsilon} f_{\epsilon}(\eta)=\frac{1}{8}\left(\frac{3}{\phi}\right)^{3 / 2} \frac{\sinh ^{2} \eta}{\cosh ^{6} \eta} .
\end{aligned}
$$

From (29) and (30) we now obtain

$$
\begin{aligned}
\frac{\nu_{T}(\zeta)}{U R} & =\frac{9}{8}\left(\frac{\phi}{3}\right)^{3 / 2} \sqrt{\zeta} \\
\frac{L_{t}(\eta, \zeta)}{R} & =8\left(\frac{\phi}{8}\right)^{3 / 2} \zeta \sqrt{\frac{\cosh ^{3} \eta}{\sinh \eta}} .
\end{aligned}
$$

From model (17), we further get

$$
\begin{aligned}
\frac{\overline{u^{\prime} w^{\prime}}(\eta, \zeta)}{U^{2}} & =-\frac{9}{8}\left(\frac{\phi}{3}\right)^{3 / 2} \frac{\alpha_{w}}{S} \frac{1}{\zeta} f_{w}^{\prime} \\
& =\frac{3}{8} \frac{1}{\zeta} \frac{\sinh \eta}{\cosh ^{3} \eta} .
\end{aligned}
$$

In a similar way, we obtain the scalar flux as

$$
\begin{aligned}
\frac{\overline{u^{\prime} c^{\prime}}(\eta, \zeta)}{U C} & =-\frac{9}{8}\left(\frac{\phi}{3}\right)^{3 / 2} \frac{\alpha_{c}}{S \sigma_{c}} \frac{1}{\zeta} f_{w}^{\prime} \\
& =\frac{1+2 \sigma_{c}}{4 \sqrt{\pi}} \frac{\Gamma\left(1 / 2+\sigma_{c}\right)}{\Gamma\left(1+\sigma_{c}\right)} \frac{1}{\zeta} \frac{\sinh \eta}{\cosh ^{1+2 \sigma_{c}} \eta}
\end{aligned}
$$

Equations (59) also give the Taylor microscale $\lambda \doteqdot$ $(10 \nu k / \epsilon)^{1 / 2}[1,2]$ as

$$
\frac{\lambda(\eta, \zeta)}{\sqrt{\nu\left(z-z_{0}\right) / U}}=\sqrt{10\left(\frac{\phi^{3} \zeta}{3 C_{\mu}}\right)^{1 / 2} \frac{\cosh ^{3} \eta}{\sinh \eta}} .
$$

Note that (64), as well as (61), diverges for $\eta=0$. The present assumptions are consequently not valid in the center part of the jet. This will be confirmed later.

Finally, it is also interesting to look at the terms of the TKE budget (25). Equations (59) allow writing the convection 
and diffusion terms $C_{k}$ and $D_{k}$ in the form of the simplified equation (33) as

$$
\begin{aligned}
& \frac{C_{k}(\eta, \zeta)}{U^{3} / R}=\frac{3}{32} \sqrt{\frac{3}{C_{\mu} \phi}} \frac{1}{\zeta^{5 / 2}}\left(3-2 \sinh ^{2} \eta\right) \frac{\sinh \eta}{\cosh ^{5} \eta} \\
& \frac{D_{k}(\eta, \zeta)}{U^{3} / R}=\frac{9}{16 \sigma_{k} \sqrt{3 C_{\mu} \phi}} \frac{1}{\zeta^{5 / 2}}\left(\sinh ^{2} \eta-2\right) \frac{\sinh \eta}{\cosh ^{5} \eta} .
\end{aligned}
$$

Recall that, with the present approximation, the (dominant) production term $P_{k}$ is assumed equal to the dissipation $\epsilon$.

In order to make all dimensionless quantities independent of the axial dimensionless coordinate $\zeta$, it is relevant to use the centerline velocity and scalar distributions, obtained from (41) and (42):

$$
\begin{aligned}
& W_{0}(\zeta) \doteqdot \bar{w}(0, \zeta)=\frac{\alpha_{w} U}{\sqrt{\zeta}} \\
& C_{0}(\zeta) \doteqdot \bar{c}(0, \zeta)=\frac{\alpha_{c} C}{\sqrt{\zeta}} .
\end{aligned}
$$

Table 1 presents a summary of our analytical formulae where $W_{0}(\zeta), C_{0}(\zeta)$, and $x^{*}(z)$ are used to make all quantities nondimensional. It should be noted that using $z-z_{0}$ in place of $x^{*}(z)$ would avoid keeping $S$ and $\delta$ in the dimensionless turbulent quantities. We made the present choice in order to keep consistent with most publications on the plane turbulent jet. Note that, in order to use only functions of $\eta$ (defined by (50)) instead of $\xi$, we used the following notation for an arbitrary function $f(\eta)$ :

$$
\begin{aligned}
& \dot{f}(\eta) \doteqdot \frac{d f}{d \eta}=\delta f^{\prime}(\xi) \\
& \widetilde{F}(\eta) \doteqdot \int_{0}^{\eta} f\left(\eta^{\prime}\right) d \eta^{\prime}=\frac{1}{\delta} F(\xi) .
\end{aligned}
$$

Table 1 presents the theoretical profiles obtained with

(i) the most general assumptions in the present theory (i.e., similarity, column \#2),

(ii) the same assumptions with production-to-dissipation equilibrium assumption (column \#3),

(iii) the previous assumptions with the classical velocity profile (41) (i.e., using the crude assumption $f_{k}^{2}=f_{\epsilon}$ in (32), column \#4, hereafter referred to as model variant \#1),

(iv) the same theory but using the empirical velocity profile (52) (column \#5, hereafter referred to as model variant \#2).

In the case of model variant \#2, the present formulae for $P_{k}, \nu_{T}$, and $\overline{u^{\prime} w^{\prime}}$ are in agreement with Agrawal and Prasad [8].

Figure 3 shows the distributions of dimensionless $k, \overline{u^{\prime} w^{\prime}}$, and $\lambda$ as a function of $\xi$. We prefer using the latter on the horizontal axis since $\eta$ is defined from $\delta$, which is not the same for the present two theoretical model variants. Moreover, this allows comparing the present models with Bradbury's [2] experiments. We can see that, despite the crude assumptions made, both models give decent predictions except for $\lambda$, where the models only catch the order of magnitude. Moreover, in the jet center part the velocity gradients become very small, breaking the production-todissipation equilibrium assumption. The TKE is consequently not well predicted by the present analytical models in this area.

The present method can also be used to give estimations of the root-mean-square of turbulent velocity fluctuations. However, the results (not given here) are not satisfactory, since our assumptions are not in agreement with a marked turbulence anisotropy.

In order to provide a validation of the proposed formulae for the terms in the TKE budget, that is, $P_{k}, C_{k}, D_{k}$, and $\epsilon$, Figure 4 shows their distributions versus $\xi$ for the two present model variants (recall that our model assumes $P_{k}=$ $\epsilon)$. It can be seen that they are in fair agreement with Bradbury's [2] experimental results. However, as previously both models fail in predicting the budget contributions (except $P_{k}$ ) in the center part of the jet (i.e., $\xi>0.5$ ), where the data obviously show that the production-to-dissipation equilibrium is broken. As a consequence of the vanishing velocity gradient on the centerline (which yields $P_{k}=0$ ), the (nonzero) dissipation is balanced by diffusion and advection in this part of the jet. Nevertheless, outside of the jet center part the different terms of the budget reproduce the measured data fairly well, including the change of sign of $C_{k}$ and $D_{k}$ around $\xi=1.2$.

Table 2 gives the model constants for the last two particular cases. They both provide similar values. The integrals of $P_{k}$, $C_{k}$, and $D_{k}$ along the transverse coordinate can be calculated analytically, and their values (displayed in the same table) are consistent with Bradbury's [2] experiments. Note that the model variant \#2, based on the empirical velocity profile (52), predicts an exactly vanishing integral of the energy diffusion.

Finally, one should mention that Bradbury's [2] experiments yield almost constant turbulent turnover timescale; that is, $k^{*} / \epsilon^{*} \approx 5.0$, where $k^{*}$ and $\epsilon^{*}$ are the dimensionless versions of $k$ and $\epsilon$ given by the left column of Table 1 . This provides a clue to further simplify (33). However, we could not get any more accurate analytical predictions with this approach.

\section{Conclusions}

We have built a consistent a complete theory of the turbulent plane jet under several assumptions: the boundary layer simplification and the self-similarity hypothesis for the velocity and concentration profiles and then the productionto-dissipation equilibrium for turbulent quantities. The last assumption can also be applied to a more accurate (but empirical) velocity profile, giving two model variants. All profiles are written in dimensionless form involving powers of the dimensionless (self-similar) transverse coordinate 


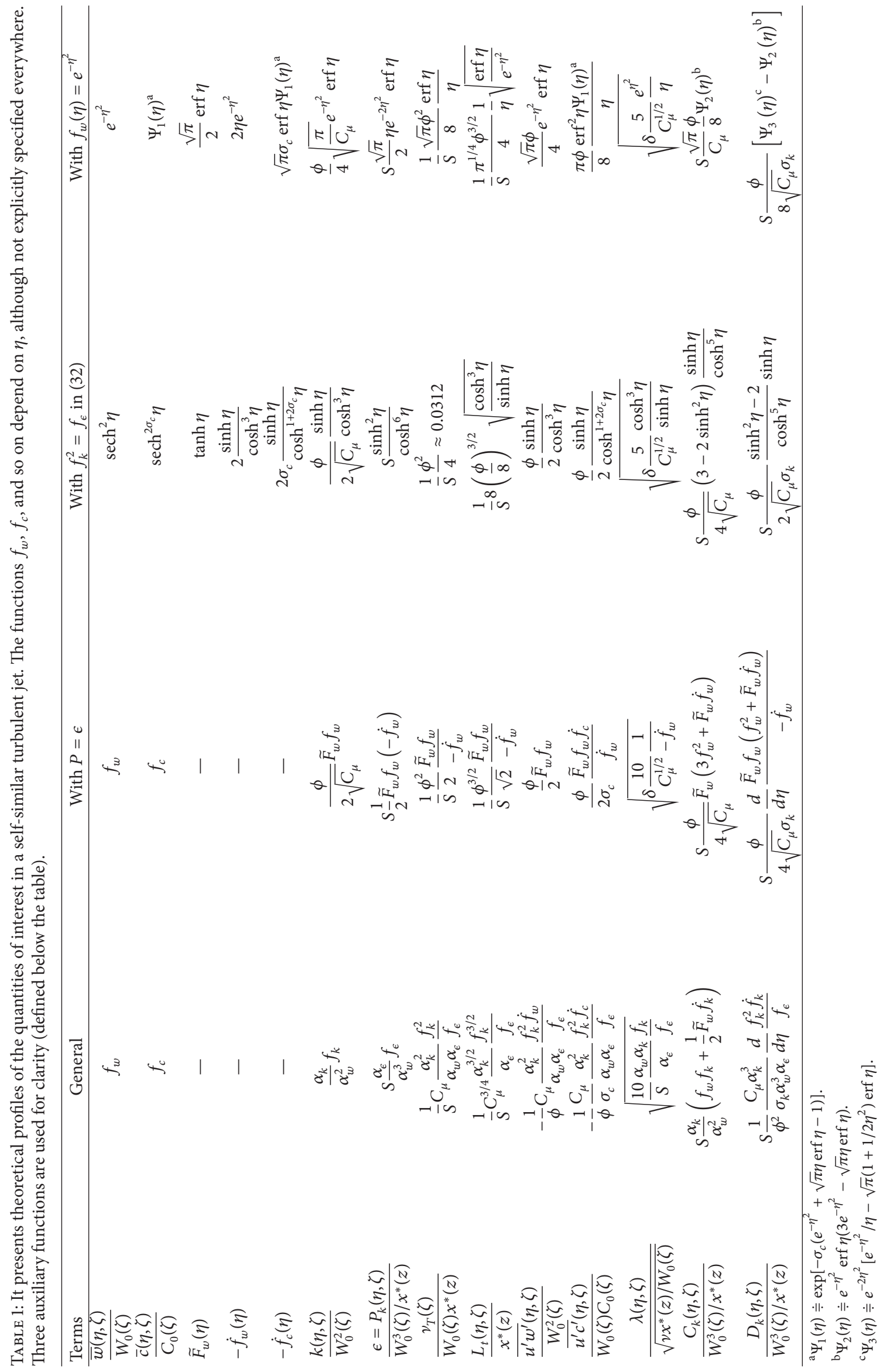




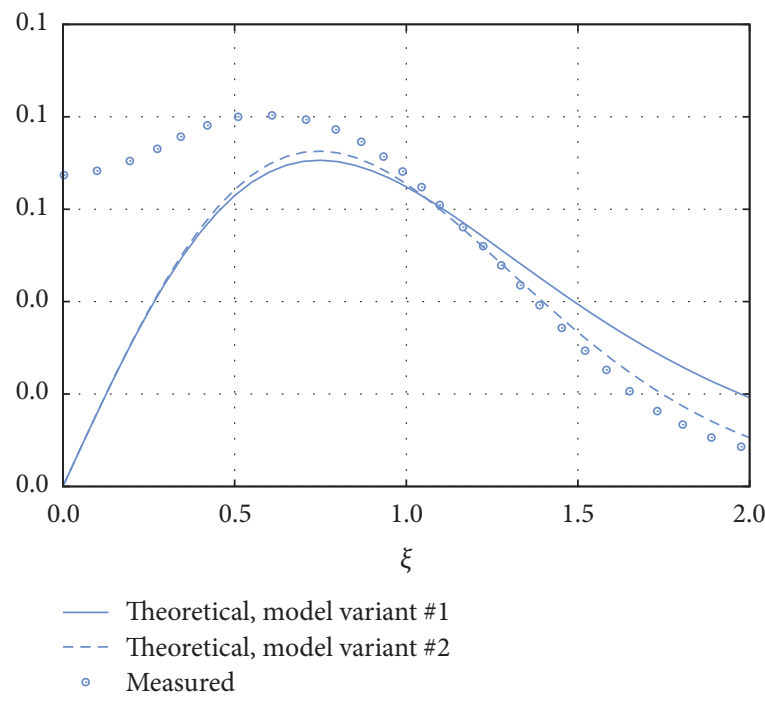

(a) TKE $k$

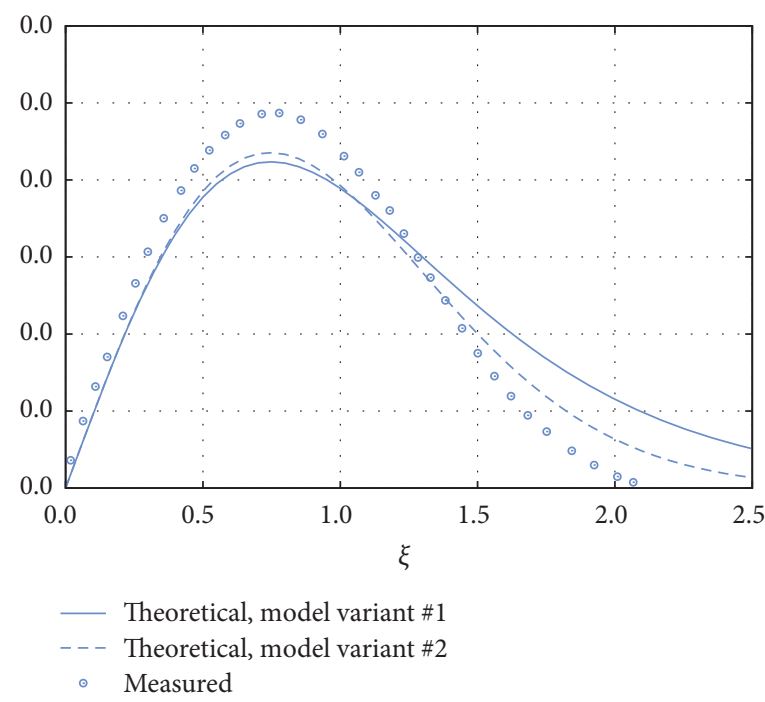

(b) Transverse Reynolds stress $\overline{u^{\prime} w^{\prime}}$

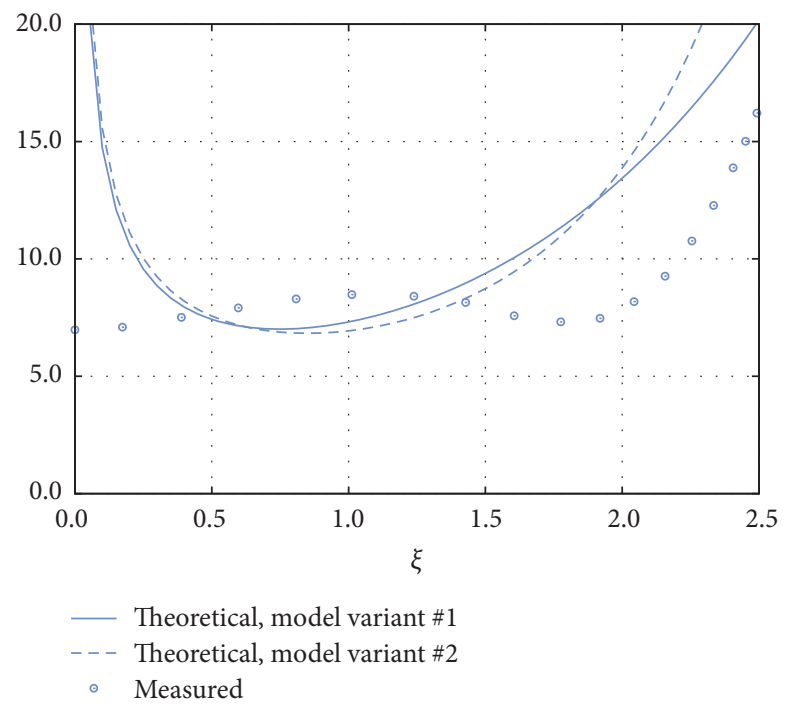

(c) Taylor microscale $\lambda$

Figure 3: Profiles of $k, \overline{u^{\prime} w^{\prime}}$, and $\lambda$. Present models \#1 (from (41), solid lines) and \#2 (from (52), dashed lines) versus measurements (symbols) from Bradbury [2]. The analytical formulae of these three quantities are given in Table 1.

and are compared to experimental data; the agreement is generally good; it is excellent for the scalar concentration but less accurate for the turbulent quantities, especially in the TKE budget, where the production-to-dissipation equilibrium fails. Integrals of the budget terms are also computed. The present work provides a synthetic approach to estimate all quantities of interest in a turbulent plane jet and can easily be extended to the round jet case of other boundary layer flows like turbulent plumes.

\section{Notation}

C: Scalar concentration scale (-)

$\bar{c}$ : Reynolds-averaged scalar concentration $(-)$ $c^{\prime}, u^{\prime}, w^{\prime}:$

$C_{0}$ :

$C_{k}$ :

$C_{\mu}:$

$D_{k}$ :

\section{$\left(\mathrm{m}^{2} \mathrm{~s}^{-3}\right)$}

$F_{w}, f_{w}, f_{c}, f_{k}, f_{\epsilon}$ : Transverse distribution functions (-)

$K: \quad$ Molecular kinematic diffusion coefficient $\left(\mathrm{m}^{2} \mathrm{~s}^{-1}\right)$

$k: \quad$ Turbulent kinetic energy $\left(\mathrm{m}^{2} \mathrm{~s}^{-2}\right)$

$L_{T}: \quad$ Integral scale (m)

$P_{k}$ : Turbulent kinetic energy production

R: $\quad$ Reynolds number (-) 
TABLE 2: Model constants and useful figures of the present models. The experimental values are from the references below the table.

\begin{tabular}{|c|c|c|c|}
\hline Constants & With $f_{k}^{2}=f_{\epsilon}$ in (32) & With $f_{w}(\eta)=e^{-\eta^{2}}$ & Experimental \\
\hline$\delta$ & $\frac{1}{\ln (1+\sqrt{2})} \approx 1.135$ & $\frac{1}{\sqrt{\ln 2}} \approx 1.201$ & - \\
\hline$\phi$ & $\approx 0.1101$ & $\approx 0.1165$ & - \\
\hline$\sigma_{c}$ & $\approx 0.63$ & $\approx 0.70$ & $0.59-0.75^{\mathrm{b}}$ \\
\hline$\alpha_{w}$ & $\frac{1}{2} \sqrt{\frac{3}{\phi}} \approx 2.610$ & $\left(\frac{2}{\pi \phi^{2}}\right)^{1 / 4} \approx 2.617$ & - \\
\hline$\alpha_{c}$ & $\frac{1+2 \sigma_{c}}{\sqrt{3 \pi \phi}} \frac{\Gamma\left(1 / 2+\sigma_{c}\right)}{\Gamma\left(1+\sigma_{c}\right)} \approx 2.325$ & $\left(\frac{\pi}{2 \phi^{2}}\right)^{1 / 4}\left[\int_{-\infty}^{+\infty} e^{-\eta^{2}} \Psi_{1}(\eta)^{\mathrm{a}} d \eta\right]^{-1} \approx 2.350$ & - \\
\hline$\frac{\alpha_{k}^{2}}{\epsilon}$ & $\frac{\sqrt{3} \phi^{3 / 2}}{8 C_{\mu}} \approx 0.0879$ & $\left(\frac{2}{\pi}\right)^{1 / 4} \frac{\phi^{3 / 2}}{4 C_{\mu}} \approx 0.0987$ & - \\
\hline$\frac{\int_{0}^{+\infty} P_{k}(\xi, \zeta) d \xi}{W_{0}^{3}(\zeta) / x^{*}(z)}$ & $\frac{2}{15} \phi \approx 0.0147$ & $\frac{1}{8} \sqrt{\frac{\pi}{3}} \phi \approx 0.0149$ & $0.0169^{c}$ \\
\hline$\frac{\int_{0}^{+\infty} C_{k}(\xi, \zeta) d \xi}{W_{0}^{3}(\zeta) / x^{*}(z)}$ & $\frac{1}{\operatorname{sqrt}_{\mu}} \frac{\phi^{2}}{16} \approx 0.00253$ & $\sqrt{\frac{2}{C_{\mu}}}\left(\tan ^{-1} \frac{\sqrt{2}}{2}\right) \frac{\phi^{2}}{16} \approx 0.00246$ & $0.0038^{\mathrm{c}}$ \\
\hline$\frac{\int_{0}^{+\infty} D_{k}(\xi, \zeta) d \xi}{W_{0}^{3}(\zeta) / x^{*}(z)}$ & $-\frac{\phi^{2}}{8 \sigma_{k} \sqrt{C_{\mu}}} \approx-0.00505$ & 0 & $0^{\mathrm{c}}$ \\
\hline
\end{tabular}

${ }^{\mathrm{a}}$ See note (a) of Table 1.

${ }^{\mathrm{b}}$ Turner [16], Mellor and Yamada [18], Sukoriansky et al. [17], Law [12], and Violeau (2009).

${ }^{\mathrm{c}}$ Bradbury [2].

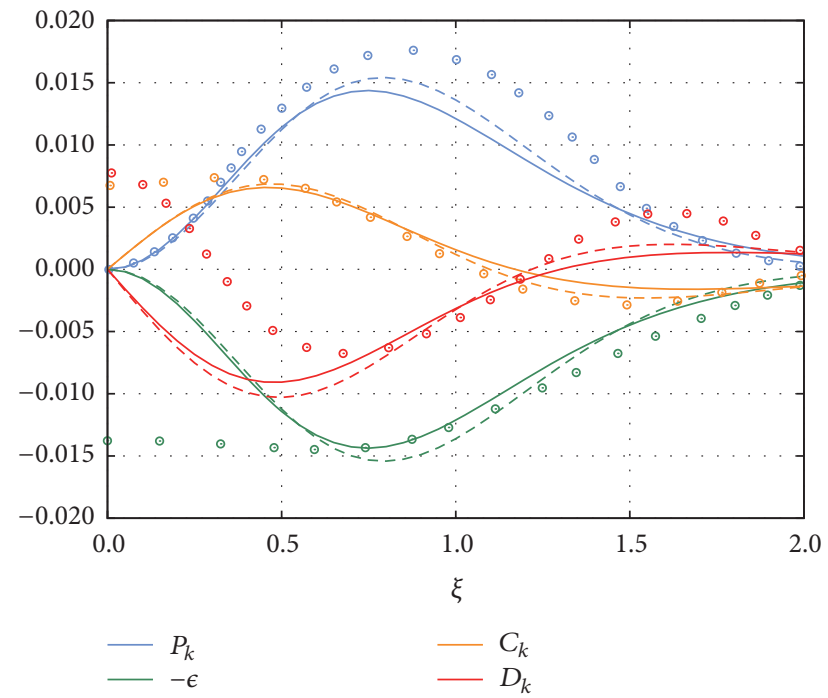

FIGURE 4: Profiles of TKE budget: production $P_{k}$ (blue), dissipation $-\epsilon$ (green), convection $C_{k}$ and diffusion $D_{k}$. Present models \#1 (from (41), solid lines) and \#2 (from (52), dashed lines) versus measurements (symbols) from Bradbury [2]. The analytical formulae of these three quantities are given in Table 1.

\footnotetext{
$R: \quad$ Size of the jet orifice $(\mathrm{m})$

S: Molecular Schmidt number (-)

$S$ : Jet slope (-)

$U$ : Velocity scale $\left(\mathrm{ms}^{-1}\right)$

$\bar{u}$ : Reynolds-averaged transverse velocity $\left(\mathrm{ms}^{-1}\right)$

$W_{0}$ : Centerline axial velocity $\left(\mathrm{ms}^{-1}\right)$

$\bar{w}$ : Reynolds-averaged axial velocity $\left(\mathrm{ms}^{-1}\right)$
}

$\begin{array}{ll}x, z: & \text { Spatial coordinates }(\mathrm{m}) \\ x^{*}: & \text { Jet half-width }(\mathrm{m}) \\ \alpha_{w}, \alpha_{c}, \alpha_{k}, \alpha_{\epsilon}: & \text { Normalising constants }(-) \\ \alpha, \gamma_{w}, \gamma_{c}: & \text { Auxiliary model constants }(-) \\ \delta, \phi: & \text { Model constants }(-) \\ \epsilon: & \text { Turbulent kinetic energy dissipation rate } \\ & \left(\mathrm{m}^{2} \mathrm{~s}^{-3}\right) \\ \lambda: & \text { Taylor microscale }(\mathrm{m}) \\ v: & \text { Molecular kinematic viscosity }\left(\mathrm{m}^{2} \mathrm{~s}^{-1}\right) \\ \nu_{T}: & \text { Turbulent kinematic viscosity }\left(\mathrm{m}^{2} \mathrm{~s}^{-1}\right) \\ \sigma_{c}: & \text { Turbulent Schmidt number }(-) \\ \sigma_{w}: & \text { 1, auxiliary notation }(-) \\ \xi, \zeta, \eta: & \text { Dimensionless coordinates }(-) .\end{array}$

\section{Conflicts of Interest}

The author declares that he has no conflicts of interest.

\section{References}

[1] S. B. Pope, Turbulent Flows, Cambridge University Press, New York, NY, USA, 2000.

[2] L. J. S. Bradbury, “The structure of a self-preserving turbulent plane jet," Journal of Fluid Mechanics, vol. 23, no. 1, pp. 31-64, 1965.

[3] A. A. Townsend, The structure of Turbulent Shear Flow, Cambridge University Press, Cambridge, UK, 1976.

[4] M. Lesieur, Turbulence in Fluids, Kluwer, Dordrecht, The Netherlands, 1990.

[5] N. E. Kotsovinos and E. J. List, "Plane turbulent buoyant jets. Part 1. Integral properties," Journal of Fluid Mechanics, vol. 81, no. 1, pp. 25-44, 1977. 
[6] C. J. Chen and W. Rodi, Vertical Turbulent Buoyant Jets. A Review of Experimental Data, Pergamon Press, Oxford, UK, 1980.

[7] J. S. Turner, “Turbulent entrainment: The development of the entrainment assumption, and its application to geophysical flows," Journal of Fluid Mechanics, vol. 173, pp. 431-471, 1986.

[8] A. Agrawal and A. K. Prasad, "Integral solution for the mean flow profiles of turbulent jets, plumes, and wakes," Journal of Fluids Engineering, vol. 125, no. 5, pp. 813-822, 2003.

[9] G. Heskestad, "Hot-wire measurements in a plane turbulent jet," Journal of Applied Mechanics, vol. 32, no. 4, pp. 721-734, 1965.

[10] E. Gutmark and I. Wygnanski, "The planar turbulent jet," Journal of Fluid Mechanics, vol. 73, no. 3, pp. 465-495, 1976.

[11] B. R. Ramapriyan and M. S. Chandrasekhara, "Measurements in vertical plane turbulent plumes," ASME Journal of Fluids Engineering, vol. 111, pp. 69-77, 1989.

[12] A. W. K. Law, "Velocity and concentration distributions of round and plane turbulent jets," Journal of Engineering Mathematics, vol. 56, no. 1, pp. 69-78, 2006.

[13] H. B. Fischer, E. J. List, J. Imberger, and N. H. Brooks, Mixing in Inland and Coastal Waters, Academic Press, New York, NY, USA, 1979.

[14] B. E. Launder and D. B. Spalding, "The numerical computation of turbulent flows," Computer Methods in Applied Mechanics and Engineering, vol. 3, no. 2, pp. 269-289, 1974.

[15] W. X. Huai and Y. H. Zeng, "Similarity solutions of vertical plane wall plume based on finite analytic method," Applied Mathematics and Mechanics, vol. 28, no. 4, pp. 447-454, 2007.

[16] J. S. Turner, Buoyancy Effects in Fluids, Cambridge University Press, Cambridge, UK, 1973.

[17] S. Sukoriansky, B. Galperin, and V. Perov, "Application of a new spectral theory of stably stratified turbulence to the atmospheric boundary layer over sea ice," Boundary-Layer Meteorology, vol. 117, no. 2, pp. 231-257, 2005.

[18] G. L. Mellor and T. Yamada, "A hierarchy of turbulence closure models for planetary boundary layers," Journal of Atmospheric Sciences, vol. 31, pp. 1791-1806, 1974. 


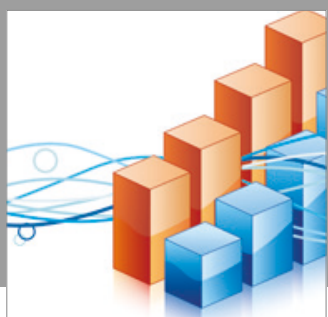

Advances in

Operations Research

vatersals

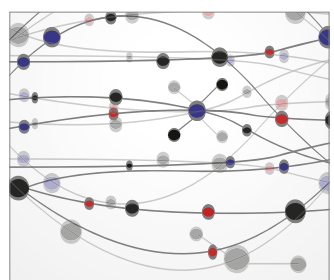

\section{The Scientific} World Journal
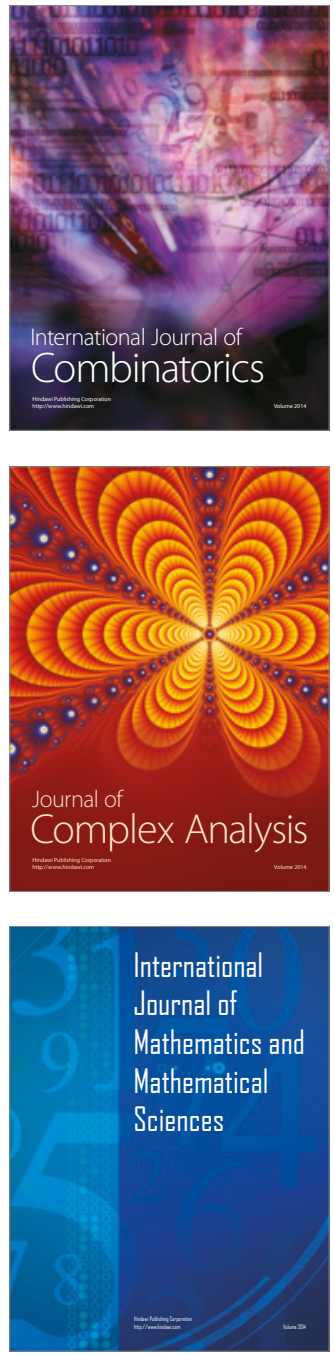
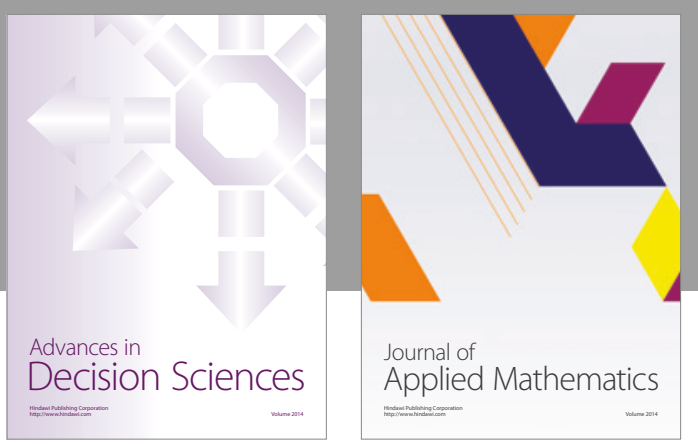

Algebra

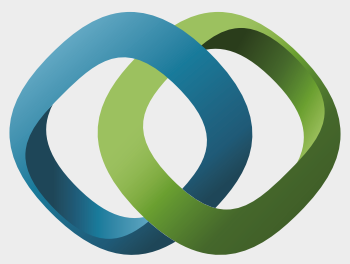

\section{Hindawi}

Submit your manuscripts at

https://www.hindawi.com
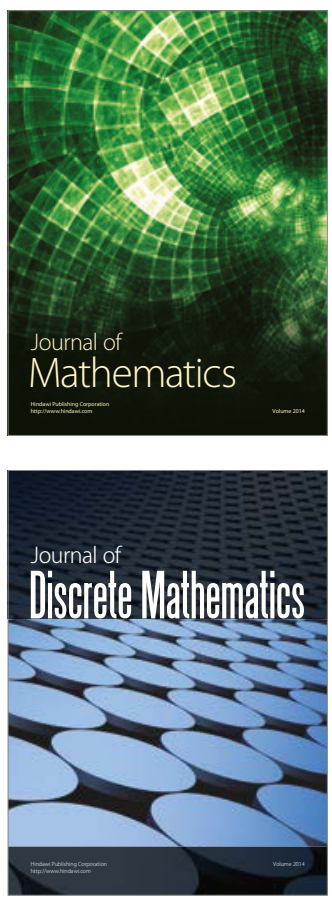

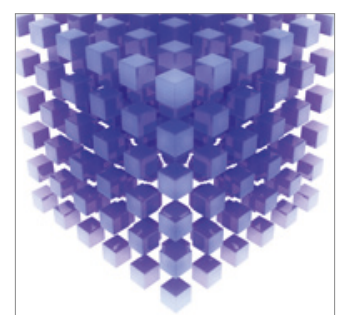

Mathematical Problems in Engineering
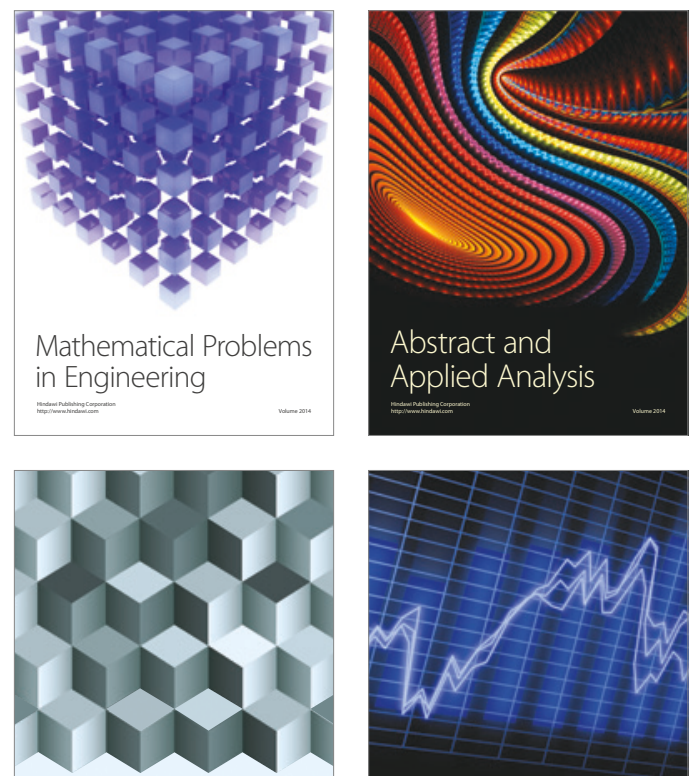

Journal of

Function Spaces

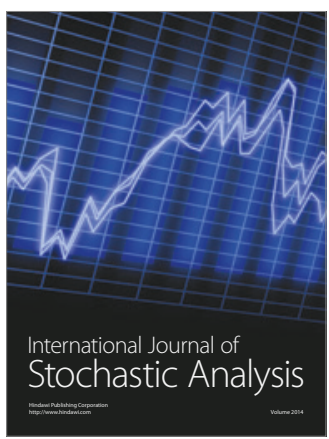

Probability and Statistics
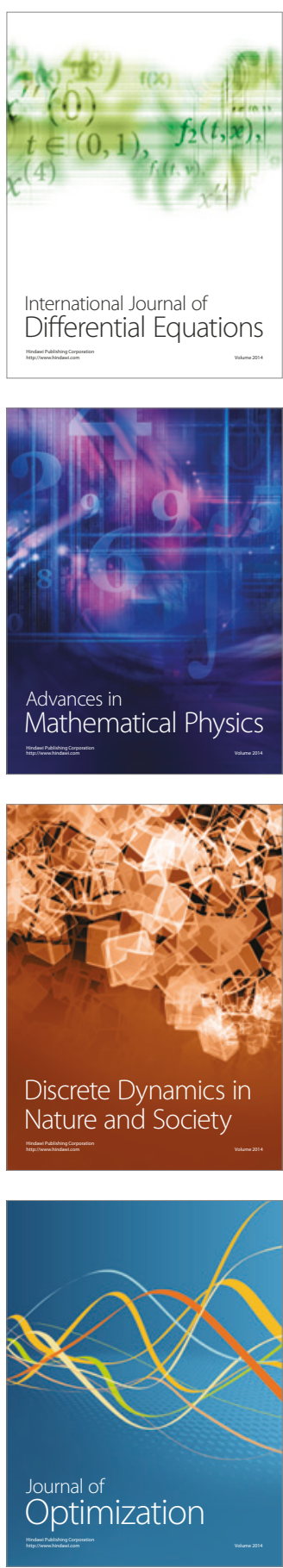